\title{
Crecimiento y supervivencia de plántulas de cinco especies de Acacia (Fabaceae), que coexisten en bosques secos neotropicales de Argentina, en distintas condiciones de disponibilidad de luz y agua
}

\author{
Paula Venier ${ }^{1}$, Marcelo Cabido ${ }^{2}$, Arnaldo Mangeaud ${ }^{3} \&$ Guillermo Funes ${ }^{2}$ \\ 1. Instituto de Fisiología y Recursos Genéticos Vegetales (CIAP-INTA). Camino 60 cuadras Km 5 11/2-(Av. 11 de setiem- \\ bre 4755), CP: X5020ICA Córdoba, Argentina; paulavenier@gmail.com \\ 2. Instituto Multidisciplinario de Biología Vegetal (UNC-CONICET). Casilla de correo 495, 5000, Córdoba, Argentina; \\ mcabido@imbiv.unc.edu.ar, gfunes@imbiv.unc.edu.ar \\ 3. Cátedra de Estadística y Biometría. Facultad de Ciencias Exactas, Físicas y Naturales. Universidad Nacional de \\ Córdoba. Av. Vélez Sarsfield 299-CP: X5000JJC, Córdoba, Argentina; amangeaud@efn.uncor.edu
}

Recibido 29-V-2012. Corregido 20-IX-2012. Aceptado 23-X-2012.

\begin{abstract}
Seedlings growth and survival of five Acacia (Fabaceae) species that coexists in neotropical semiarid forests of Argentina, under different light and water availability conditions. Seedling establishment is one of the most risky stages of plants, especially in arid and semiarid regions, where low water availability and high solar radiation influence its emergence, development and survival. In seasonally dry xerophytic forests occurring in North-Western Córdoba, central Argentina, five neotropical species of Acacia co-exist: A. aroma, A. caven, A. atramentaria, A. gilliesii and A. praecox. With the aim to evaluate growth variables and survival of these five species seedlings, in response to water stress and different light availability conditions, a greenhouse experiment was undertaken from March to June of 2010. Although small differences were found between species $(\mathrm{F}=5.66, \mathrm{p}=0.001)$, all of them showed high percentages of seedling survival in response to different light and water treatments, suggesting that seedlings would be tolerant to water stress and could be established both in light and shade. On the other hand, although all species showed an increase in growth in light conditions and without water stress, we have found some trends towards a greater growth in the seedlings of $A$. aroma, A. caven and $A$. atramentaria when compared to those of $A$. praecox and A. gilliessi in most of the variables considered $(\mathrm{F}=41.9, \mathrm{p}<0.0001 ; \mathrm{F}=7.06, \mathrm{p}<0.0001 ; \mathrm{F}=53.59, \mathrm{p}<0.0001)$. This pattern was confirmed through a cluster analysis that classified the species in two main groups. These results, together with others already reported, would indicate a regenerative niche differentiation that might be favoring the regional coexistence of these five species in semiarid forests in central Argentina. Rev. Biol. Trop. 61 (2): 501-514. Epub 2013 June 01.
\end{abstract}

Key words: Acacia, seedling, shade, water stress, coexistence, semiarid woodland, Argentina.

Le emergencia y establecimiento de la plántula son etapas muy críticas en el ciclo de vida de las plantas (Harper 1977), especialmente en las zonas áridas y semiáridas donde la baja disponibilidad de agua suele ser limitante (Noy-Meir 1973). La luz es otro de los factores ambientales que influyen sobre la emergencia, desarrollo, establecimiento y supervivencia de las plántulas (Sack 2004, Sánchez-Gómez et al. 2006b, Sánchez-Gómez et al. 2008, Quero et al. 2008).
El estudio de caracteres relacionados con la tolerancia de las plántulas a la disponibilidad de luz y agua ha recibido especial atención, ya que pueden ocurrir efectos interactivos entre esos dos factores. Ambos, se presentan en la naturaleza de forma heterogénea y su influencia sobre el desarrollo y establecimiento de las plántulas ha sido muy estudiada, principalmente en bosques de climas tropicales y en matorrales mediterráneos (Poorter et al. 2005, Sánchez-Gómez et al. 2006a, Quero et 
al. 2008, Sánchez-Gómez et al. 2008). Se han encontrado distintos compromisos o trade-offs entre la tolerancia a la sombra y la tolerancia a la sequía en muchas especies leñosas, en especial de sistemas mediterráneos (Sack 2004, Sánchez-Gómez et al. 2008). De esta manera, la utilización diferencial de la luz y el agua por parte de las diferentes especies podría favorecer su coexistencia tanto en el espacio como en el tiempo (Sack 2004, Sánchez-Gómez et al. 2006a), especialmente en especies que son morfo-funcionalmente similares en la etapa adulta (Grubb 1977).

La reducción en la cantidad y calidad de luz (R/RL) así como en la disponibilidad de agua en el suelo, puede repercutir fuertemente en el desarrollo y supervivencia de la plántula, disminuyendo en muchas ocasiones la biomasa tanto aérea como subterránea, aunque esta respuesta puede ser especie-específica (Kitajima \& Fenner 2000, Sack 2004, Gómez-Aparicio et al. 2006, Villagra \& Cavagnaro 2006).

En bosques subtropicales estacionales del centro de Argentina, como los bosques xerófilos del Chaco Occidental de la Provincia de Córdoba, se ha encontrado que algunas especies leñosas se establecen preferentemente debajo de las copas de los árboles debido a las condiciones favorables que encuentran las plántulas bajo sombra, al resguardo de las altas temperaturas y de la radiación directa (Páez \& Marco 2000, Barchuck et al. 2005). En tales sistemas, las plantas están expuestas a períodos de sequía en invierno debido a la falta de lluvias. No obstante, deben soportar un déficit hídrico también en verano, debido a que, si bien esa es la estación más húmeda del año, las temperaturas extremas, junto a la alta radiación solar (Venier 2011), aumentan la evaporación del agua del suelo generando un estrés hídrico estival para las plantas (Noy-Meir 1985).

Entre las especies que habitan estos bosques, coexisten cinco del género Acacia: $A$. aroma Guilles ex Hook. \& Arn., A. caven (Molina) Molina, A. atramentaria Benth., presentes preferentemente en sitios perturbados o abiertos junto a $A$. gilliesii Steud. y $A$. praecox Griseb frecuentes en sitios conservados o cerrados. Si bien estas cinco especies son en su etapa adulta muy similares en términos de caracteres vegetativos (Díaz et al. 1998), trabajos previos han demostrado que existen diferencias marcadas entre ellas en características de la cubierta seminal (Venier et al. 2012a) y en algunas respuestas de la germinación a diversos factores (Funes \& Venier 2006, Funes et al. 2009, Venier 2011, Venier et al. 2012b). No obstante, aún falta conocer si difieren en caracteres propios de la etapa de plántula. Los sitios abiertos y cerrados que conforman el mosaico de bosques xerófilos del noroeste de Córdoba -producto de distintos niveles de perturbación antrópica- (Cabido et al. 1994) varían en las condiciones ambientales (Venier 2011). Por lo tanto, cabría esperar diferencias en la capacidad de establecimiento de las plántulas que pudieran facilitar la coexistencia de estas especies congéneres que viven en simpatría en los bosques heterogéneos del noroeste de la provincia de Córdoba.

Muchos de los estudios que evalúan la interacción entre los factores luz y agua sobre las plántulas se refieren a especies de climas mediterráneos (Sánchez-Gómez et al. 2006a, Quero et al. 2008, Sánchez-Gómez et al. 2008). Mientras que, hasta lo que conocemos, no existen estudios que evalúen las respuestas de las plántulas a los efectos combinados de disponibilidad de luz y agua, en especies congéneres neotropicales de zonas semiáridas. Por lo tanto, el objetivo de este trabajo fue determinar, mediante un experimento controlado, la capacidad de supervivencia y crecimiento de las plántulas de cinco especies neotropicales de Acacia, en diferentes condiciones de disponibilidad de luz y de agua. Por otro lado, diferencias interespecíficas en la susceptibilidad de las plántulas a amenazas físicas y bióticas durante el establecimiento pueden generar una diferenciación en el nicho regenerativo facilitando la coexistencia de las especies (Dalling \& Hubbell 2002). Por lo tanto, a partir de una combinación de análisis univariados y multivariados, se buscó comprender el patrón general de las respuestas de las plántulas de estas cinco especies a factores ambientales, con el 
propósito de contribuir a explicar su coexistencia en los bosques xerófilos de la Provincia de Córdoba, en el centro de Argentina.

\section{MATERIALES Y MÉTODOS}

Área de estudio y especies: El área de estudio corresponde a los bosques xerófilos estacionales del noroeste de la provincia de Córdoba, en el centro de Argentina, pertenecientes al Distrito de los Llanos (Ragonese \& Castiglioni 1970) o Chaco Árido (Morello et al. 1985), dentro de la Provincia Fitogeográfica Chaqueña (con coordenadas $30^{\circ} 45^{\prime} 32^{\prime}$ ' S - 64'55'30" W en su punto central) (Cabrera 1976). La precipitación media anual es de $500 \mathrm{~mm}$, principalmente concentrada en la estación cálida (entre noviembre y marzo), y una temperatura media anual de $19^{\circ} \mathrm{C}$ (Zak et al. 2008).

En la actualidad estos bosques se componen de un mosaico de comunidades en diferente estado sucesional (Cabido et al. 1994, Zak \& Cabido 2002). Dentro de los parches de bosque maduro (con alta cobertura de leñosas y canopia cerrada), es frecuente la presencia de A. praecox y A. gilliesii, mientras que en los matorrales abiertos, con escasos emergentes arbóreos y diferentes niveles de perturbación, prosperan $A$. aroma, $A$. caven y $A$. atramentaria (Cabido et al. 1994, Zak \& Cabido 2002).

Estimación de la supervivencia y crecimiento de plántulas en invernadero: Para cada especie se utilizaron 120 semillas recolectadas de frutos maduros de al menos diez individuos en el noroeste de la Provincia de Córdoba. Debido a que tres de las especies presentan dormición física - $A$. aroma, $A$. caven y A. atramentaria- (Funes \& Venier 2006, Venier et al. 2012a), las semillas de las cinco especies fueron escarificadas con papel de lija y luego se incubaron en cámara de germinación a $25 / 15^{\circ} \mathrm{C} 12 / 12 \mathrm{hr}$ luz/oscuridad-temperatura óptima de germinación de las cinco especies(Funes \& Venier 2006, Funes et al. 2009). Una vez emergida la radícula, las semillas fueron trasladadas al invernadero, sembradas cada una en un recipiente de plástico negro de $30 \mathrm{~cm}$ de largo por $5 \mathrm{~cm}$ de diámetro, rellenados con un sustrato de tierra rica en materia orgánica y perlita (mineral de origen volcánico) en una proporción 3:1. Cada recipiente fue previamente humedecido alcanzando al comienzo del experimento un nivel de humedad promedio del $23.7 \%$ según registros realizados sobre el sustrato de recipientes controles utilizando el dispositivo Moisture Probe Meter -MPM160-B-. Este contenido de humedad inicial tanto para el tratamiento de luz como de sombra, correspondió a un potencial hídrico de -0.71MPa obtenido a través de un psicrómetro (Wescor C-52 Sample Chamber-HR-33T-Dew Point Microvoltmeter). Las 120 semillas de cada especie fueron distribuidas en tres réplicas de luz y tres de sombra, y regadas manualmente con agua de grifo. Cada réplica consistió de 20 recipientes de los cuales diez fueron sometidos a estrés hídrico, y los diez restantes fueron mantenidos a capacidad de campo a través del riego periódico durante los 105 días que duró el experimento.

Tratamiento estrés hídrico: Una vez emergida la plántula, entendiendo como tal a la planta desde el momento de apertura de los cotiledones hasta lo que duró el experimento (Barchuck et al. 2006), aquellas correspondientes al tratamiento de estrés hídrico dejaron de ser regadas, no recibiendo agua hasta el final del experimento (Quero et al. 2006). Además, cada condición de agua estuvo sometida a las distintas condiciones de luz (SánchezGómez 2007).

Con el propósito de obtener un registro periódico del nivel de estrés hídrico al que fueron sometidas las plántulas, se midió semanalmente la humedad del sustrato de los recipientes controles (cuatro en sombra y cinco en luz), con el dispositivo Moisture Probe Meter-MPM-160-B, el cual registra el contenido volumétrico de agua que presenta el sustrato (volumetric water content, VWC) (Quero et al. 2006). Al final del experimento se registraron valores de humedad del sustrato cercanos al $0 \%$ en la condición luz y $2.8 \%$ en 
la condición sombra. Estos valores se corresponden con potenciales hídricos de $-28.44 \mathrm{MPa}$ y $-19.46 \mathrm{MPa}$, respectivamente, medidos a partir de un psicrómetro (Wescor C-52 Sample Chamber-HR-33T-Dew Point Microvoltmeter).

Tratamiento luz y sombra: Se crearon tres réplicas de luz y tres de sombra. La condición de sombra fue generada cubriendo las macetas con dos telas tipo "media sombra" (Battaglia et al. 2000, Sánchez-Gómez et al. 2008) consiguiendo sobre las plántulas una radiación fotosintéticamente activa promedio (RFA-medida con el dispositivo LICOR LI $1400-)$ a lo largo del día de $29.46 \pm 17 \mu \mathrm{mol} /$ $\mathrm{m}^{2}$.s (un 7\% de la luz promedio incidente en el invernadero en el horario de mayor insolación). Para la condición de luz, las plántulas contaron con la luz natural del día alcanzada en el invernadero, la cual resultó en promedio de $262.68 \pm 121 \mu \mathrm{mol} / \mathrm{m}^{2}$.s (un $63 \%$ de la luz promedio incidente en el invernadero en el horario de mayor insolación). Durante los 105 días que duró el experimento las plántulas fueron mantenidas en el invernadero a la temperatura controlada de $25 \pm 2^{\circ} \mathrm{C}$.

Variables medidas: Al final del experimento se registró el porcentaje de supervivencia de las plántulas en las distintas condiciones de disponibilidad de luz y agua. Además, se midieron variables de crecimiento considerando en este caso a cada plántula una réplica: la altura de la plántula (desde la base del tallo -superficie del sustrato- hasta la yema foliar más alta), la longitud de la raíz, la biomasa aérea (tallo y hojas) y subterránea (raíces), y la biomasa total (Reich et al. 1998, de Villalobos \& Peláez 2001). Para la determinación de la biomasa aérea y subterránea, se consideró el peso seco $(\mathrm{g})$ de la parte aérea y subterránea de cada plántula, luego de haber sido almacenadas en estufa a $60^{\circ} \mathrm{C}$ durante $72 \mathrm{hr}$ (Villagra \& Cavagnaro 2006). Luego se calculó la relación entre inversión de biomasa en la parte aérea y subterránea de la plántula de cada especie (biomasa aérea/biomasa subterránea $-\mathrm{g} / \mathrm{g}-$ ) y para cada tratamiento (Villagra \& Cavagnaro 2000, Sánchez-Gómez et al. 2008).

Para comparar el porcentaje de supervivencia, la longitud de la raíz y la altura de la plántula entre las especies, se realizó un Análisis de la Varianza trifactorial con Rangos, puesto que las variables no cumplieron con los supuestos de normalidad ni homogeneidad de varianzas, ni aún cuando se intentó transformar los datos a arcoseno (para el caso de los porcentajes de supervivencia) o logaritmo (Conover \& Iman 1981, Zar 1999, Urcelay et al. 2003). La interpretación de los resultados que arroja este tipo de ANAVA, se realiza de la misma manera que para un ANAVA paramétrico (Zar 1999, Urcelay et al. 2003).

Se analizó la inversión de biomasa de cada especie en la parte aérea y subterránea de la plántula a través de la relación biomasa aérea/ biomasa subterránea $(\mathrm{g} / \mathrm{g})$. Se comparó tal relación entre las especies por medio de un Análisis de la Varianza trifactorial con Rangos (Conover \& Iman 1981). El mismo análisis se utilizó para comparar la biomasa total de las plántulas entre las especies.

En cada uno de los análisis, los factores fueron: Especie, Sombra y Estrés hídrico. Para las variables en que la interacción trifactorial fue significativa se realizó un test a posteriori LSD Fisher cuyos resultados se muestran en el apéndice 1.

Luego de analizar el comportamiento individual de las variables, se realizó un Análisis de Conglomerados para evaluar el patrón multivariado en las respuestas de las plántulas a las distintas condiciones de disponibilidad de luz y agua, de manera tal que se pudiera determinar el patrón general de similitud entre las especies de Acacia teniendo en cuenta todos los caracteres conjuntamente (Crisci \& López-Armengol 1983). Para la construcción del dendrograma se utilizaron las siguientes 20 variables: porcentaje de supervivencia en cada tratamiento (cuatro variables), altura de la plántula y longitud de la raíz en cada tratamiento (ocho variables), biomasa total en cada tratamiento (cuatro variables), y relación biomasa aérea/biomasa subterránea ante cada 
tratamiento (cuatro variables). Se utilizó la técnica "ligamiento promedio aritmético no ponderado" (Unweighted Paired Group Method of Arithmetic Averages -UPGMA-) (Sneath \& Sokal 1973). Para visualizar grupos diferentes, la línea de corte se realizó con el 95\% de la variabilidad acumulada (medida como distancia euclídea) según Infostat 2007 (Di Rienzo et al. 1999). Para realizar los análisis, se utilizaron los programas estadísticos Infostat 2007 (Di Rienzo et al. 1999) y SPSS 15.0 (2006).

\section{RESULTADOS}

Supervivencia de las plántulas: Sólo el factor Especie y la interacción Sombra*Estrés Hídrico resultaron significativos $(\mathrm{F}=5.66$, $\mathrm{p}=0.001 ; \mathrm{F}=9.2, \mathrm{p}=0.0042$, respectivamente), mientras que la interacción triple entre los factores Especie*Sombra*Estrés Hídrico no resultó significativa $(\mathrm{F}=1.18, \mathrm{p}=0.334)$.

$\mathrm{Si}$ bien las cinco especies mostraron altos porcentajes de supervivencia en los distintos tratamientos (entre un $65 \%$ y un $100 \%$, Fig. 1), se destaca $A$. praecox obteniendo entre el 95 y $100 \%$ de supervivencia en todos los tratamientos. Acacia atramentaria mostró los menores porcentajes de supervivencia en los tratamientos de sombra con y sin estrés hídrico, mientras que $A$. aroma obtuvo el menor porcentaje de supervivencia en luz con estrés hídrico.

Altura de la plántula: Los tres factores analizados (Especies, Sombra y Estrés Hídrico) y sus interacciones resultaron significativos (Cuadro 1). Aunque todas las especies alcanzaron mayor altura con luz sin estrés hídrico y menor altura en sombra con estrés hídrico (Fig. 2), A. caven alcanzó la mayor altura promedio y $A$. praecox la menor (Fig. 2). Asimismo $A$. praecox no varió significativamente entre los distintos tratamientos; y A. aroma mostró una marcada disminución de la altura de la plántula en sombra con estrés hídrico (Fig. 2).

Longitud de la raíz: Los factores Especie, Sombra y su interacción, además de la interacción de los factores Sombra*Estrés Hídrico y Especie*Sombra*Estrés Hídrico resultaron significativos (Cuadro 1).

Aunque en general todas las especies alcanzaron raíces más largas creciendo en luz, con escasa variación dentro del tratamiento de estrés hídrico (con y sin estrés hídrico), $A$. aroma, A. caven y A. atramentaria fueron las

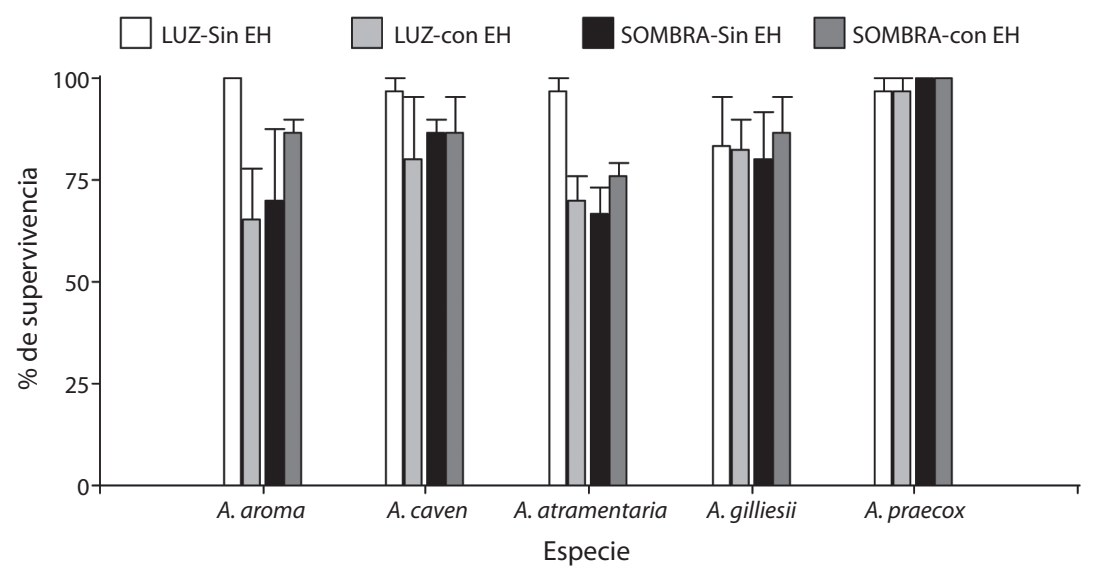

Fig. 1. Porcentajes promedios $( \pm \mathrm{EE})$ de supervivencia de plántulas, en luz y sombra, con y sin estrés hídrico, para cada una de las especies de Acacia. EH=Estrés Hídrico.

Fig. 1. Mean percentages $( \pm \mathrm{SE})$ of seedlings survival in light and shade, with and without water stress for each species of Acacia. $\mathrm{EH}=$ Water Stress. 


\section{CUADRO 1}

Resultados del ANAVA trifactorial con rangos correspondientes a los efectos de los factores "especies", "estrés hídrico", "sombra" y sus interacciones, sobre la altura de la plántula y la longitud de la raíz $(\mathrm{p} \leq 0.05$ corresponde a resultados estadísticamente significativos)

TABLE 1

Results of three-factor ANOVA with ranks corresponding to the effects of the factors "species", "water stress",

"shade" and their interactions on height and length seedling ( $\mathrm{p} \leq 0.05$ indicates statistically significant results)

\begin{tabular}{lcccccc}
\multicolumn{1}{c}{ Fuente de variación } & \multicolumn{3}{c}{ Altura de la plántula } & \multicolumn{2}{c}{ Longitud de la raíz } \\
& $\mathrm{gl}$ & $\mathrm{F}$ & $\mathrm{p}$-valor & $\mathrm{gl}$ & $\mathrm{F}$ & $\mathrm{p}$-valor \\
Especie & 4 & 41.9 & $<0.0001$ & 4 & 5.53 & 0.0002 \\
Sombra & 1 & 91.58 & $<0.0001$ & 1 & 624.48 & $<0.0001$ \\
Estrés Hídrico & 1 & 83.01 & $<0.0001$ & 1 & 3.4 & 0.0659 \\
Especie*Sombra & 4 & 20.28 & $<0.0001$ & 4 & 17.17 & $<0.0001$ \\
Especie*Estrés Hídrico & 4 & 3.75 & 0.0051 & 4 & 2.08 & 0.0817 \\
Sombra*Estrés Hídrico & 1 & 19.14 & $<0.0001$ & 1 & 9.27 & 0.0025 \\
Especie*Sombra*Estrés Hídrico & 4 & 2.88 & 0.0224 & 4 & 3.33 & 0.0104 \\
\hline
\end{tabular}
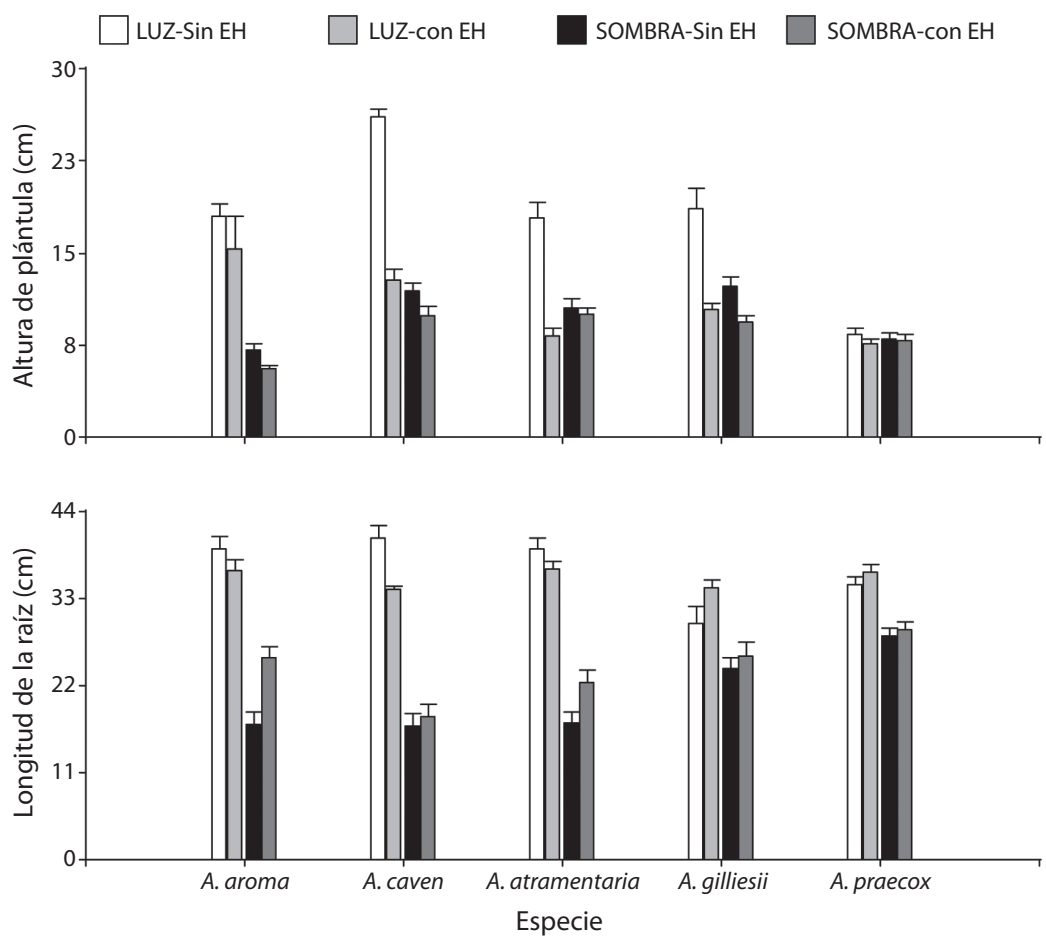

Fig. 2. Altura promedio de la plántula y longitud promedio de la raíz ( $\pm \mathrm{EE}$ ) para cada especie de Acacia en luz y sombra, con y sin estrés hídrico. EH=Estrés Hídrico.

Fig. 2. Mean height of the seedling and mean root length $( \pm \mathrm{SE})$ for each species of Acacia in light and shade, with and without water stress. EH=Water Stress. 
que presentaron las mayores longitudes (Fig. 2). Asimismo estas tres especies fueron las de menor crecimiento radicular cuando estuvieron a la sombra y sin estrés hídrico (Fig. 2).

Biomasa total: Los tres factores analizados (Especie, Sombra, Estrés Hídrico) y la interacción entre ellos resultaron significativos (Cuadro 2).

Si bien las plántulas de las cinco especies tuvieron mayor biomasa cuando crecieron con luz que bajo sombra, y además la producción de biomasa fue mayor con luz sin estrés hídrico que con estrés hídrico, A. aroma, A. caven y $A$. atramentaria fueron las de mayor crecimiento, y A. praecox presentó la menor producción de biomasa total. En sombra, las cinco especies no difirieron en la producción de biomasa total y además el crecimiento fue similar entre las distintas condiciones de disponibilidad de agua (Fig. 3).

\section{Relación biomasa aérea/subterránea:}

Los tres factores analizados y sus interacciones resultaron significativos (Cuadro 2).

Con disponibilidad de luz, con y sin estrés hídrico, en general todas las especies, invirtieron biomasa de manera similar entre la parte aérea y la subterránea (ya que los valores fueron cercanos a 1). No obstante, sólo $A$. gilliesii mostró mayor inversión en la parte aérea que en la subterránea, mientras que $A$. aroma y $A$. atramentaria presentaron mayor biomasa subterránea cuando estuvieron con estrés hídrico (valores menores a 1) (Fig. 3).

En cambio a la sombra, aunque todas las especies invirtieron más biomasa en la parte aérea (valores mayores a 1) que en la subterránea, en el caso de $A$. aroma, $A$. caven y $A$. atramentaria la inversión fue mayor en la parte aérea cuando estuvieron sin estrés hídrico, mientras que en $A$. gilliesii y $A$. praecox la inversión fue similar dentro del tratamiento de estrés hídrico (con y sin estrés hídrico). Asimismo en $A$. aroma, A. caven y A. atramentaria, la relación entre biomasa aérea y biomasa subterránea fue mayor que en $A$. gilliesii y $A$. praecox, siendo esta última la especie que presentó menor relación (Fig. 3).

El análisis conjunto de las respuestas de las plántulas a las distintas condiciones de disponibilidad de luz y agua permitió distinguir a través del dendrograma, dos grupos principales de especies según la similitud entre ellas considerando todas las variables medidas (Fig. 4). Uno comprendido por A. aroma, A. caven y A. atramentaria, y el otro conformado por $A$. gilliesii y A. praecox (Fig. 4).

\section{CUADRO 2}

Resultados del ANAVA trifactorial con rangos correspondientes a los efectos de los factores "especies", "estrés hídrico", "sombra" y sus interacciones, sobre la biomasa total de las plántulas y la relación biomasa aérea/biomasa subterránea $(\mathrm{p}<0.05$ corresponde a resultados estadísticamente significativos)

TABLE 2

Results of three-factor ANOVA with ranks corresponding to the effects of the factors "species", "water stress", "shade" and their interactions, on total biomass of seedlings and on the ratio aerial biomass/belowrground biomass ( $\mathrm{p} \leq 0.05$ indicates statistically significant results)

\begin{tabular}{lcccccc}
\multicolumn{1}{c}{ Fuente de variación } & \multicolumn{4}{c}{ Biomasa Total } & \multicolumn{2}{c}{ Relación Biomasa Aérea/Biomasa Subterránea } \\
& gl & F & p-valor & gl & F & p-valor \\
Especie & 4 & 7.06 & $<0.0001$ & 4 & 53.59 & $<0.0001$ \\
Sombra & 1 & 1717.82 & $<0.0001$ & 1 & 2529.15 & $<0.0001$ \\
Estrés Hídrico & 1 & 69.73 & $<0.0001$ & 1 & 46.1 & $<0.0001$ \\
Especie*Sombra & 4 & 14.33 & $<0.0001$ & 4 & 100.09 & $<0.0001$ \\
Especie*Estrés Hídrico & 4 & 2.82 & 0.0247 & 4 & 8.76 & $<0.0001$ \\
Sombra*Estrés Hídrico & 1 & 16.92 & $<0.0001$ & 1 & 10.34 & 0.0014 \\
Especie*Sombra*Estrés Hídrico & 4 & 4.19 & 0.0024 & 4 & 3.03 & 0.0173 \\
\hline
\end{tabular}



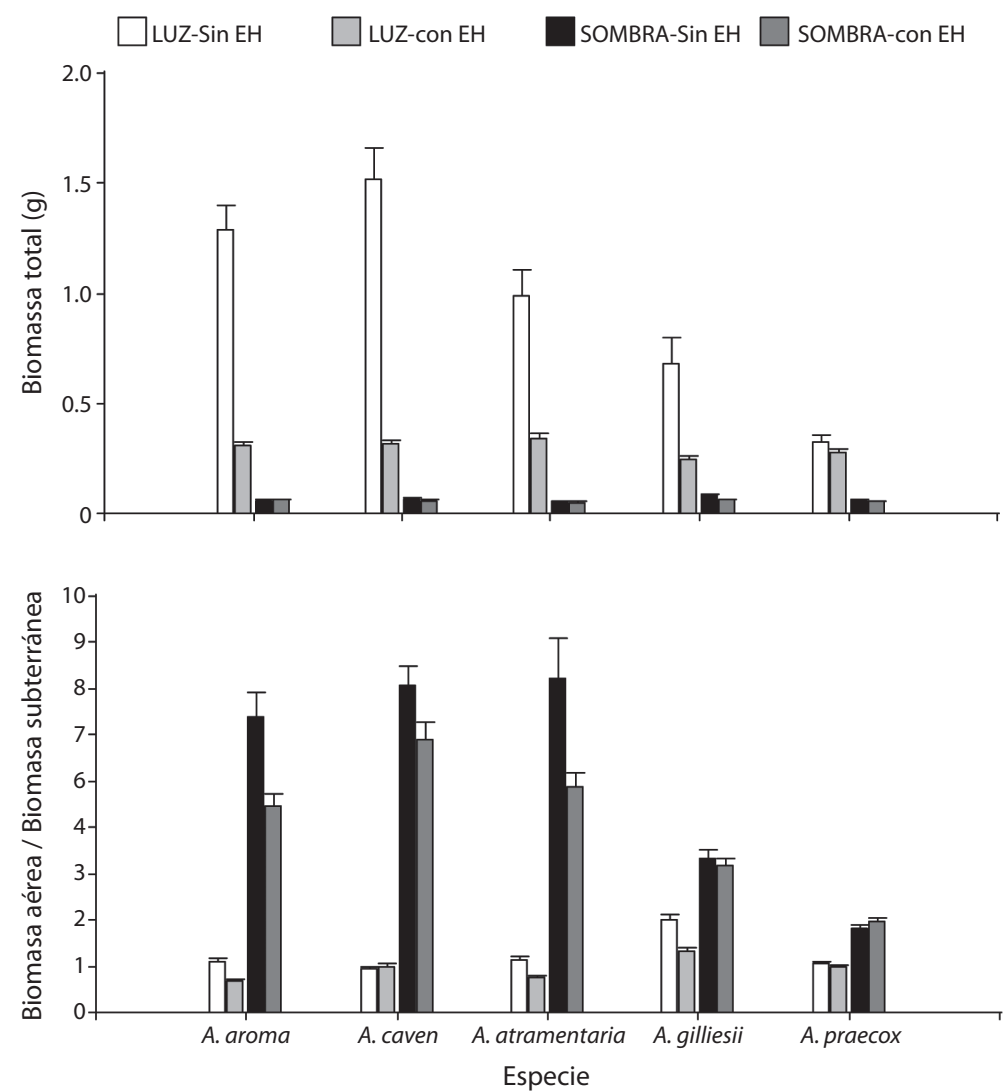

Fig. 3. Biomasa total promedio $( \pm \mathrm{EE})$ y relación entre biomasa aérea y subterránea (biomasa aérea/biomasa subterráneag/g-) ( \pm EE) de las plántulas de cada especie de Acacia en luz y sombra, con y sin estrés hídrico. EH=Estrés Hídrico.

Fig. 3. Mean total biomass $( \pm \mathrm{SE})$ and aerial and belowground biomass ratio (aerial biomass/belowground biomass-g/g) $( \pm \mathrm{SE})$ of seedlings of each species of Acacia in light and shade, with and without water stress. EH=Water Stress.

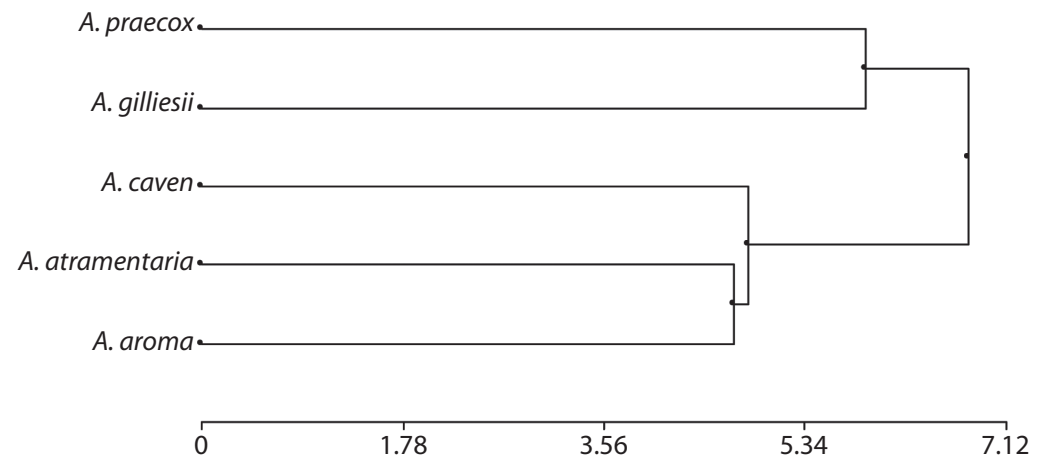

Fig. 4. Dendrograma. Agrupación de las especies de Acacia según la similitud entre ellas en las respuestas de las plántulas a distintas condiciones de luz y agua. Correlación Cofenética $=0.954$.

Fig. 4. Dendrogram. Grouping of Acacia species by the similarity between them in the responses of seedlings to different light and water conditions. Cophenetic correlation $=0.954$. 


\section{DISCUSIÓN}

Los altos porcentajes de supervivencia de las plántulas obtenidos en las cinco especies de Acacia en todos los tratamientos realizados, sugieren que estas especies no tendrían fuertes restricciones de los factores analizados para su establecimiento, al menos en condiciones de laboratorio. Sin embargo, cabe destacar algunas diferencias observadas. Por ejemplo, A. praecox mostró en general, los mayores porcentajes de supervivencia, en especial en los tratamientos de reducción de luz con y sin estrés hídrico. En los bosques estudiados, esta especie se encuentra muy asociada al interior de los mismos (Cabido et al. 1994) en donde se observa una reducción de la cantidad y calidad de luz (relación rojo: rojo lejano) que llega al suelo (Zalazar et al. 2009). Por otra parte, los elevados porcentajes de supervivencia de las cinco especies de Acacia en condiciones de estrés hídrico, indicarían que podrían soportar durante la temprana etapa de establecimiento de las plántulas, el déficit de agua existente en el Chaco Seco del centro de Argentina.

En general, todas las especies crecieron menos a la sombra que con disponibilidad de luz (reflejado en la menor altura de las plántulas, menor longitud de las raíces y menor biomasa total). Tal disminución en el crecimiento puede deberse a la importante reducción de radiación fotosintéticamente activa que existe a la sombra (Holmgren et al. 1997). Además, tanto $A$. caven como A. aroma se encuentran fuertemente asociadas a parches de vegetación abiertos en donde la cantidad de radiación fotosintéticamente activa (RFA) no es una limitante como en el interior del bosque (Venier 2011). Asimismo, a la sombra, el estrés hídrico tendría menor impacto sobre las plántulas que en condiciones de disponibilidad de luz, ya que los individuos de las distintas especies alcanzaron un crecimiento similar con déficit de agua y $\sin$ estrés hídrico. Posiblemente, las condiciones de sombra alivian el efecto de la escasez de agua, o al menos no agravan el impacto del estrés hídrico sobre las plántulas, tal como se ha mostrado en otras especies leñosas (Sánchez-Gómez et al. 2006a, Quero et al. 2008), en parte porque se reducen las demandas de evapotranspiración de las plántulas (Holmgren et al. 1997). Sin embargo, el crecimiento de la mayoría de las especies se vio afectado por el estrés hídrico cuando sus plántulas crecieron con disponibilidad de luz, disminuyendo el crecimiento principalmente en $A$. caven, $A$. atramentaria y $A$. gilliesii; mientras que $A$. praecox sería la especie menos influenciada por el estrés hídrico. Resultados similares se registraron para especies leñosas de sistemas mediterráneos, en donde se encontró que las condiciones de sequía redujeron significativamente la producción de biomasa ante condiciones de mayor intensidad de luz (Sack 2004).

En general, las plántulas de las cinco especies tuvieron en promedio raíces de mayor longitud que la altura de la parte aérea, relación que fue más marcada en $A$. praecox. Diversos autores han reportado que los arbustos que crecen en zonas áridas y semiáridas tienden a tener largas raíces para poder acceder al agua de las capas más profundas del suelo (Noy-Meir 1973, Gutiérrez \& Squeo 2004), y aumentar así la posibilidad de captura de agua. De esta manera, la mayor longitud de la raíz en relación al tallo, resultaría ventajoso para las plántulas, permitiéndoles sobrevivir en ambientes secos (Lloret et al. 1999). Por lo tanto, nuestro resultado podría estar también relacionado con las características de escasa humedad del suelo de la región en dónde habitan las especies estudiadas.

Un aspecto a destacar es que A. aroma, $A$. caven y $A$. atramentaria presentaron mayor biomasa total que $A$. gilliesii y $A$. praecox cuando crecieron con luz. La alta acumulación de biomasa le otorgaría cierta ventaja competitiva a estas especies, permitiéndoles colonizar rápidamente suelos desnudos en sitios de bosque abierto. Este resultado se relacionaría con la condición de "cicatrizadoras" de los bosques xerófilos perturbados de Córdoba, con que son conocidas estas especies, principalmente A. aroma y A. caven (Demaio et al. 2002, Zak \& Cabido 2002). Al mismo tiempo, sería 
contrastante con los patrones de establecimientos de muchas plántulas en los sistemas áridos y semiáridos que son mediados por un proceso de facilitación bajo el efecto nodriza de plantas adultas (Flores \& Jurado 2003).

Por último, la relación entre biomasa aérea y biomasa subterránea fue mayor en sombra que con disponibilidad de luz, para las cinco especies, con la mayor relación en A. aroma, A. caven y A. atramentaria. Este resultado indicaría que durante los primeros meses de vida, las plántulas de estas especies invertirían más biomasa en tallo y hojas que en raíces cuando están a la sombra, coincidiendo con resultados reportados por otros autores para especies leñosas del hemisferio norte (Reich et al. 1998), pero en desacuerdo con Sánchez-Gómez (2007), quien reportó como una estrategia de tolerancia a la sombra, el uso conservador de los recursos a través de una baja relación biomasa aérea/biomasa subterránea.

En conclusión, si bien las cinco especies de Acacia crecen mejor en condiciones de disponibilidad de luz y sin estrés hídrico, existen diferencias entre ellas que muestran una tendencia hacia un mayor crecimiento en las plántulas de $A$. aroma, $A$. caven y $A$. atramentaria que en las de $A$. praecox y $A$. gilliessi en la mayoría de las variables y tratamientos considerados. Estas diferencias se evidencian en el análisis de conglomerados, en donde se incorporaron todas las variables en su conjunto, las de crecimiento y supervivencia, mostrando dos grupos bien definidos. Por un lado, A. aroma, A. caven y $A$. atramentaria, especies que en general se encuentran asociadas a sitios abiertos, con cierto grado de perturbación antrópica, y por otro A. gilliessi y $A$. praecox, especies asociadas a bosques cerrados, bien conservados (Cabido et al. 1994, Zak \& Cabido 2002). Venier et al. (2012a) encontraron diferencias entre las especies en distintas características de la cubierta seminal, tales como espesor de los tejidos seminales y presencia de dormición física, lo cual también permitió agruparlas de la misma manera que en este trabajo. La diferenciación en estas variables es importante ya que pueden originar distintos patrones de germinación entre las especies (Wang et al. 2009).

Los resultados obtenidos por Venier et al. (2012a), sumados a diferencias interespecíficas encontradas en respuestas germinativas (Venier 2011), junto a los observados en el presente estudio, podrían indicar la existencia de posibles diferencias en el nicho regenerativo de las especies congéneres estudiadas. De esta manera, los caracteres regenerativos podrían estar favoreciendo la coexistencia regional de las cinco especies de Acacia en los bosques xerófilos del centro de Argentina (Grubb 1977, Silvertown 2004, Pérez-Ramos et al. 2010).

Por último, teniendo en cuenta la alta tasa de deforestación que vienen sufriendo los bosques de Córdoba debido principalmente a la expansión de la agricultura (Grau et al. 2005, Zak et al. 2008, Hoyos et al. 2012), la información obtenida en este estudio podría ser de utilidad para considerarla en planes de manejo y restauración con especies nativas en zonas áridas. Particularmente, las tres especies de Acacia que mostraron mayor crecimiento en condiciones de luz, podrían ser consideradas para recuperar sitios que actualmente presentan grandes superficies de suelo desnudo.

\section{AGRADECIMIENTOS}

Este trabajo fue financiado por aportes parciales de las siguientes instituciones: Consejo Nacional de Investigaciones Científicas y Técnicas (CONICET-PIP 01286 y 6196/05), Secretaría de Ciencia y Tecnología (SECYT), de la Universidad de Córdoba, el Inter-American Institute for Global Change Research (IAI) CRNII-2005, el FONCyT (ANPCyT), y el Ministerio de Ciencia y Tecnología de la Provincia de Córdoba (Préstamo BID-PID No. 013/2009 y PIO 2012). Agradecemos a tres revisores anónimos y al editor por sus valiosos comentarios y sugerencias que seguramente han incrementado la calidad del manuscrito. 


\section{RESUMEN}

El establecimiento de la plántula es una de las etapas más riesgosas para las plantas, especialmente en zonas áridas y semiáridas donde la sequía y alta radiación solar influyen sobre su emergencia, desarrollo y supervivencia. Se evaluó en invernadero la supervivencia y variables de crecimiento en plántulas sometidas a estrés hídrico y a distintas condiciones de luz, en cinco especies de Acacia (A. aroma, A. caven, A. atramentaria, A. gilliesii y A. praecox) que coexisten en los bosques xerófilos de Córdoba, Argentina. Aunque se encontraron diferencias entre las especies $(\mathrm{F}=5.66, \mathrm{p}=0.001)$, todas tuvieron altos porcentajes de supervivencia en las distintas condiciones de luz y agua, sugiriendo que serían tolerantes al estrés hídrico y podrían establecerse bajo luz o sombra. Si bien todas las especies mejoraron el crecimiento con luz y sin estrés hídrico, A. aroma, A. caven y A. atramentaria mostraron una tendencia hacia un mayor crecimiento en la mayoría de las variables consideradas $(\mathrm{F}=41.9, \mathrm{p}<0.0001 ; \mathrm{F}=7.06$, $\mathrm{p}<0.0001 ; \mathrm{F}=53.59, \mathrm{p}<0.0001)$. Estos resultados sumados a otros ya reportados indicarían una diferenciación de nichos regenerativos favoreciendo la coexistencia regional de estas especies en los bosques xerófilos de Córdoba.

Palabras clave: Acacia, plántula, sombra, estrés hídrico, coexistencia, bosque semiárido, Argentina.

\section{REFERENCIAS}

Barchuck, A.H., A. Valiente-Banuet \& M.P. Díaz. 2005. Effect of shrubs and seasonal variability of rainfall on the establishment of Aspidosperma quebrachoblanco in two edaphically contrasting environments. Austral Ecol. 30: 695-705.

Barchuck, A.H., E.B. Campos, C. Oviedo \& M.P. Díaz. 2006. Supervivencia y crecimiento de plántulas de especies leñosas del Chaco Árido sometidas a remoción de la biomasa aérea. Ecol. Austral 16: 47-61.

Battaglia, L.L., S.A. Foré \& R.R. Sharitz. 2000. Seedling emergence, survival and size in relation to light and water availability in two bottomland hardwood species. J. Ecol. 88: 1041-1050.

Cabido, M., A. Manzur, L. Carranza \& C. GonzálezAlbarracin. 1994. La vegetación y el medio físico del Chaco Árido en la provincia de Córdoba, Argentina Central. Phytocoenología 24: 423-460.

Cabrera, A. 1976. Regiones Fitogeográficas de Argentina. Enciclopedia Argentina de Agricultura y Jardinería. ACME, Buenos Aires, Argentina.

Conover, W.J. \& R.L. Iman. 1981. Rank transformations as a bridge between parametric and nonparametric statistics. Am. Stat. 35: 124-129.

Crisci, J.V. \& M.F. López-Armengol. 1983. Introducción a la teoría y práctica de la taxonomía numérica. Cátedra de Introducción a la Taxonomía. Facultad de Ciencias Naturales. Universidad Nacional de La Plata, La Plata, Argentina.

Dalling, J.W. \& S.P. Hubbell. 2002. Seed size, growth rate and gap microsite conditions as determinants of recruitment success for pioneer species. J. Ecol. 90: 557-568.

de Villalobos, A.E. \& D.V. Peláez. 2001. Influences of temperature and water stress on germination and establishment of Prosopis caldenia Burk. J. Arid Environ. 49: 321-328.

Demaio, P., U.O. Karlin \& M. Medina. 2002. Árboles Nativos del Centro de Argentina. Lola, Buenos Aires, Argentina.

Díaz, S., M. Cabido \& F. Casanoves. 1998. Plant functional and environmental filters at a regional scale. J. Vegetation Sci. 9: 113-122.

Di Rienzo, J., W. Robledo, F. Casanoves, M. Balzarini, L. González, A.W. Guzmán \& E.M. Tablada. 1999. Infostat. Versión Beta. Estadística y Biometría, Facultad de Ciencias Agropecuarias, Universidad Nacional de Córdoba, Córdoba, Argentina.

Flores, J. \& E. Jurado. 2003. Are nurse-protégé interactions more common among plants from arid environments? J. Vegetation Sci. 14: 911-916.

Funes, G., S. Díaz \& P. Venier. 2009. La temperatura como principal determinante de la germinación en especies del Chaco seco de Argentina. Ecol. Austral 19: 129-138.

Funes, G. \& P. Venier. 2006. Dormancy and germination in three Acacia (Fabaceae) species from central Argentina. Seed Sci. Res. 16: 77-82.

Gómez-Aparicio, L., F. Valladares \& R. Zamora. 2006. Differential Light responses of Mediterranean tree saplings: linking ecophysiology with regeneration niche in tour co-occurring species. Tree Physiol. 26: 947-958.

Grau, H.R., N.I. Gasparini \& T.M. Aide. 2005. Agriculture expansion and deforestation in seasonally dry forests of north-west Argentina. Environ. Conserv. 32: $140-148$.

Grubb, P.J. 1977. The maintenance of species-richness in plant communities: the importance of the regeneration niche. Biol. Rev. 52: 107-145.

Gutiérrez, J.R. \& F.A. Squeo. 2004. Importancia de los arbustos en los ecosistemas semiáridos de Chile. Ecosistemas 13: 36-45.

Harper, J. 1977. Population Biology of Plant. Academic, Londres, Inglaterra.

Holmgren, M., M. Scheffer \& M.A. Huston. 1997. The intreplay of facilitation and competition in plant communities. Ecology 78: 1966-1975. 
Hoyos, L., A.M. Cingolani, M.R. Zak, M.V. Vaieretti, D.E. Gorla \& M.R. Cabido. 2012. Deforestation and precipitation patterns in the arid Chaco forests of central Argentina. Appl. Veg. Sci. DOI: 10.1111/j.1654-109X.2012.01218.X

Kitajima, K. \& M. Fenner. 2000. Ecology of Seedling Regeration en Seeds: The Ecology of Regeneration in Plant Communities. CAB International, Wallinford, Oxan, Reino Unido.

Lloret, F., C. Casanovas \& J. Peñuelas. 1999. Seedling survival of Mediterranean shrubland species in relation to root: shoot ratio, seed size and water and nitrogen use. Funct. Ecol. 13: 210-216.

Morello, J.H., C. Protomastro, L. Sancholuz \& C. Blanco. 1985. Estudio macroecológico de los Llanos de La Rioja. Serie del cincuentenario de la Administración de Parques Nacionales $\mathrm{N}^{\circ}$ 5. Administración de Parques Nacionales Buenos Aires, Argentina.

Noy-Meir, I. 1973. Desert Ecosystems: Environment and producers. Annu. Rev. Ecol. Syst. 4: 25-51.

Noy-Meir, I. 1985. Desert ecosystem structure and function, p. 92-103. In M. Evenari, I. Noy-Meir \& D. Goodall (eds.). Ecosystems of the World: Hot Deserts and Arid Shrublands. Elsevier, Amsterdam, Holanda.

Páez, S.A. \& D.E. Marco. 2000. Seedling habitat structure in dry Chaco forest (Argentina). J. Arid Environ. 46: 57-68.

Pérez-Ramos, I.M., L. Gómez-Aparicio, R. Villar, L.V. García \& T. Marañón. 2010. Seedling growth and morphology of three oak species along field resource gradients and seed mass variation: a seedling agedependent response. J. Vegetation Sc. 21: 419-437.

Poorter, L., F. Bongers, F.J. Sterck \& H. Wöll. 2005. Beyond the regeneration phase: differentiation of height-light trajectories among tropical tree species. J. Ecol. 93: 256-267.

Quero, J.L., R. Villar, T. Marañón \& R. Zamora. 2006. Interactions of drought and shade effects on seedlings of four Quercus species: physiological and structural leaf responses. New Phytol. 170: 819-839.

Quero, J.L., R. Villar, T. Marañón, A. Murillo \& R. Zamora. 2008. Respuesta plástica a la luz y al agua en cuatro especies mediterráneas del género Quercus (Fagaceae). Rev. Chil. Hist. Nat. 81: 373-385.

Ragonese, A. \& J. Castiglione. 1970. La vegetación del Parque Chaqueño. Bol. Soc. Arg. Bot. 11: 133-160.

Reich, P.B., M.G. Tjoelker, M.B. Walters, D.W. Vanderklein \& C. Buschena. 1998. Close association of RGR, leaf and root morphology, seed mass and shade tolerance in seedlings of nine boreal tree species grown in high and low light. Funct. Ecol. 12: $327-338$.

Sack, L. 2004. Responses of temperate woody seedlings to shade and drought: do trade-offs limit potenctial niche differentiation? Oikos 107: 110-127.

Sánchez-Gómez, D., F. Valladares \& M.A. Zabala. 2006a. Performance of seedlings of Mediterranean woody species under experimental gradients of irradiance and water availability: trade.offs and evidence for niche differentiation. New Phytol. 170: 795-806.

Sánchez-Gómez, D., M.A. Zabala \& F. Valladares. 2006b. Seedling survival responses to irradiance are differentially influenced by low-water availability in four tree species of the Iberian cool temperate-Mediterranean ecotone. Acta Oecol. 30: 322-332.

Sánchez-Gómez, D. 2007. Respuesta de plántulas leñosas mediterráneas a la disponibilidad de luz y agua en condiciones experimentales. Ecosistemas 16: 119-123.

Sánchez-Gómez, D., M.A. Zavala \& F. Valladares. 2008. Functional traits and plasticity linked to seedlings' performance under shade and drought in Mediterranean woody species. Ann. For. Sci. 65: 311.

Silvertown, J. 2004. Plant coexistence and the niche. Trends Ecol. Evol. 19: 605-611.

Sneath, P.H.A. \& R.R. Sokal. 1973. Numerical Taxonomy. The Principles and Practise of Numerical Classification. Freeman, San Francisco, California, EE.UU.

SPSS. 2006. SPSS Versión 15.0. IBM SPSS Statistics, Chicago, EE.UU.

Urcelay, C., M.S. Bret-Harte, S. Díaz \& F.S. Chapin. 2003. Mycorrhizal colonization mediated by species interactions in Arctic tundra. Oecologia 137: 399-404.

Venier, P. 2011. ¿Pueden los caracteres regenerativos explicar la coexistencia de especies de Acacia (Fabaceae) en los bosques xerófilos de la Provincia de Córdoba? Tesis de Doctorado, Universidad Nacional de Córdoba, Córdoba, Argentina.

Venier, P., G. Funes \& C. Carrizo-García. 2012a. Physical dormancy and histological features of seeds of five Acacia species (Fabaceae) from xerophytic forests of central Argentina. Flora 207: 39-46.

Venier, P., C. Carrizo-García, M. Cabido \& G. Funes. 2012b. Survival and germination of three hard-seeded Acacia species after simulated cattle ingestion: The importance of the seed coat structure. S. Afr. J. Bot. 79: $19-24$.

Villagra, P.E. \& J.B. Cavagnaro. 2000. Effects of clayish and sandy soils on the growth of Prosopis argentina and P. alpataco seedlings. Ecol. Austral 10: 113-121. 
Villagra, P.E. \& J.B. Cavagnaro. 2006. Water stress effects on the seedling growth of Prosopis argentina and Prosopis alpataco. J. Arid Environ. 64: 390-400.

Wang, J.H., C.C. Baskin, X.L. Cui \& G.Z. Du. 2009. Effect of phylogeny, life history and habitat correlates on seed germination of 69 arid and semiarid zone species from northwest China. Evol. Ecol. 23: 827-846.

Zak, M. \& M. Cabido. 2002. Spatial patterns of the Chaco vegetation of central Argentina: Integration of remote sensing and phytosociology. Appl. Veg. Sci. 5: 213-226.
Zak, M.R., M. Cabido, D. Cáceres \& S. Díaz. 2008. What drives accelerated land cover change in central Argentina? Synergistic consequences of climatic, socioeconomic, and technological factors. Environ. Manag. 42: 181-189.

Zalazar, M., G. Funes \& M.P. Venier. 2009. Factores que afectan la germinación de Justicia squarrosa Griseb, forrajera nativa de la región chaqueña de la Argentina. Agriscientia 26: 1-6.

Zar, J.H. 1999. Biostatistical Analysis. Prentice Hall, New Jersey, EE.UU. 


\section{APÉNDICE 1}

Resultados del test a posteriori LSD Fisher para la interacción entre los factores Especie*Estrés Hídrico*Sombra sobre la altura de la plántula, la longitud de la raíz, la biomasa total y la relación biomasa aérea/biomasa subterránea. Todas las variables están expresadas en rangos. Diferentes letras indican diferencias estadísticamente diferentes $(\mathrm{p}<0.05)$

\section{APPENDIX 1}

Results of a post-hoc LSD Fisher test for the interaction between the factors Species*Water Stress*Shade on the seedling height, root length, total biomass and aerial biomass/belowground biomass ratio. All variables are expressed as ranks.

Different letters indicate statistically different $(\mathrm{p}<0.05)$

\begin{tabular}{|c|c|c|c|c|c|c|c|c|}
\hline \multirow{2}{*}{$\begin{array}{l}\text { Especie -Estrés Hídrico - } \\
\text { Luz/Sombra } \\
\text { A. aroma }\end{array}$} & \multicolumn{2}{|c|}{$\begin{array}{l}\text { Altura de la plántula } \\
\text { (rango) }\end{array}$} & \multicolumn{2}{|c|}{$\begin{array}{l}\text { Longitud de la raíz } \\
\qquad \text { (rango) }\end{array}$} & \multicolumn{2}{|c|}{$\begin{array}{l}\text { Biomasa total } \\
\quad(\text { rango) }\end{array}$} & \multicolumn{2}{|c|}{$\begin{array}{c}\text { Relación biomasa aérea/ } \\
\text { biomasa subterránea (rango) }\end{array}$} \\
\hline & & & & & & & & \\
\hline Luz-Con EH & 273.63 & e $f g h$ & 356.5 & hi & 343.39 & $\mathrm{fgh}$ & 49 & $\mathrm{a}$ \\
\hline Luz-Sin EH & 392.4 & $\mathrm{i}$ & 392.02 & $\mathrm{i}$ & 449.22 & $\mathrm{i} \mathrm{j}$ & 143.47 & $\mathrm{c}$ \\
\hline Sombra-Con EH & 67.19 & $\mathrm{a}$ & 172.4 & $\mathrm{c} \mathrm{d} \mathrm{e}$ & 129.63 & $\mathrm{bcd}$ & 398.19 & g \\
\hline Sombra-Sin EH & 126.83 & $a b$ & 79.64 & $\mathrm{a}$ & 128.07 & $\mathrm{bcd}$ & 445.14 & $\mathrm{~h} \mathrm{i}$ \\
\hline \multicolumn{9}{|l|}{ A. caven } \\
\hline Luz-Con EH & 316.58 & $\mathrm{~h}$ & 332.98 & $\mathrm{~h}$ & 345.85 & $\mathrm{~g} \mathrm{~h}$ & 117 & $\mathrm{bc}$ \\
\hline Luz-Sin EH & 478.91 & $\mathrm{j}$ & 405.31 & $\mathrm{i}$ & 468.55 & $\mathrm{j}$ & 109.43 & $\mathrm{~b}$ \\
\hline Sombra-Con EH & 233.29 & $\mathrm{de}$ & 97.02 & $\mathrm{ab}$ & 122.15 & $\mathrm{bcd}$ & 438.98 & $\mathrm{~h} \mathrm{i}$ \\
\hline Sombra-Sin EH & 300.85 & $\mathrm{fgh}$ & 83 & $\mathrm{a}$ & 155.04 & $\mathrm{de}$ & 461.54 & $\mathrm{i}$ \\
\hline \multicolumn{9}{|l|}{ A. atramentaria } \\
\hline Luz-Con EH & 179.95 & $\mathrm{bcd}$ & 368.95 & h i & 359.36 & $\mathrm{~g} \mathrm{~h}$ & 69.86 & a \\
\hline Luz-Sin EH & 387 & $\mathrm{i}$ & 395.16 & $\mathrm{i}$ & 430.14 & $\mathrm{i}$ & 140.95 & $\mathrm{c}$ \\
\hline Sombra-Con EH & 243.27 & $\mathrm{~d} \mathrm{f}$ & 137.8 & $\mathrm{bc}$ & 82.68 & $\mathrm{a}$ & 411.98 & $\mathrm{~g} \mathrm{~h}$ \\
\hline Sombra-Sin EH & 246.3 & e $f g$ & 79.13 & a & 93.5 & $a b$ & 449.78 & $\mathrm{i}$ \\
\hline \multicolumn{9}{|l|}{ A. gilliesii } \\
\hline Luz-Con EH & 257.69 & e $f g h$ & 327.69 & $\mathrm{~h}$ & 307.58 & $\mathrm{f}$ & 192.58 & d \\
\hline Luz-Sin EH & 376.54 & $\mathrm{i}$ & 274.44 & $\mathrm{~g}$ & 364.66 & $\mathrm{~h}$ & 271.44 & e \\
\hline Sombra-Con EH & 220.6 & $\mathrm{c} \mathrm{de}$ & 192.37 & $d$ e $f$ & 125.35 & $\mathrm{bcd}$ & 351.02 & $\mathrm{f}$ \\
\hline Sombra-Sin EH & 306.28 & $\mathrm{~g} \mathrm{~h}$ & 160.87 & $\mathrm{~cd}$ & 182.72 & e & 356.3 & $\mathrm{f}$ \\
\hline \multicolumn{9}{|l|}{ A. praecox } \\
\hline Luz-Con EH & 145.6 & $\mathrm{~b}$ & 364.95 & $\mathrm{~h} \mathrm{i}$ & 326.59 & $f g$ & 120.22 & $\mathrm{bc}$ \\
\hline Luz-Sin EH & 176.14 & $\mathrm{bc}$ & 334.91 & $\mathrm{~h}$ & 340.48 & $\mathrm{fgh}$ & 131.53 & $\mathrm{bc}$ \\
\hline Sombra-Con EH & 163.52 & $\mathrm{~b}$ & 223.77 & $\mathrm{f}$ & 113.88 & $\mathrm{abc}$ & 275.63 & $\mathrm{e}$ \\
\hline Sombra-Sin EH & 171.52 & $\mathrm{bc}$ & 214.08 & $\mathrm{df}$ & 140.25 & $\mathrm{~cd}$ & 251.7 & $\mathrm{e}$ \\
\hline $\begin{array}{l}\text { Interacción Especie- } \\
\text { Estrés Hídrico-Sombra }\end{array}$ & \multicolumn{2}{|c|}{$\mathrm{F}=2.88 \mathrm{p}=0.0224$} & \multicolumn{2}{|c|}{$\mathrm{F}=3.33 \mathrm{p}=0.0104$} & \multicolumn{2}{|c|}{$\mathrm{F}=4.19 \mathrm{p}=0.0024$} & \multicolumn{2}{|c|}{$\mathrm{F}=3.03 \mathrm{p}=0.0173$} \\
\hline
\end{tabular}

